\title{
NÂNG CAO Độ CHÍNH XÁC KHI ÚNG DỤNG CÔNG NGHỆ RTK, TRONG THÀNH LẬP BẢN ĐỒ SỐ TỶ LỆ LỚN
}

\author{
HOÀNG TH!̣ THỦY \\ Đại học Mỏ Địa Chất
}

\section{Tóm tắt:}

Nội dung nghiên cúu bao gồm khảo sát độ chính xác đo chi tiết thành lập bản đồ số tỷ lệ lớn bằng công nghệ RTK, PPK, CORS thông qua kết quả thưc nghiệm. Kết hợp đo GPS tại một số điểm khống chế cùng với đo chi tiết để nâng cao độ chính xác trong thành lập bản đồ số tỷ lệ lớn. Xây dụng modul chuơng trình hiệu chỉnh tọa độ các điểm đo chi tiết dụa vào các điểm đo GPS theo bài toán biến đổi tọa độ. Kết quả nghiên cưu cho thấy, tính hiệu quả và đảm bảo yêu cầu độ chính xác trong công tác thành lập bản đồ số tỷ lệ lớn khi sử dụng công nghệ RTK, PPK, CORS cùng với điểm đo $G P S$

\section{Mở đầu:}

Công nghệ GPS (Global Positioning System) đã được phát triển rộng khắp trong các ngành, đặc biệt trong công tác trắc địa. Việc nghiên cứu ứng dụng công nghệ GPS thành lập bản đồ số tỷ lệ lớn đem lại kết quả cao. Phương pháp đo tương đối động thơi gian thực (RTK) và phương pháp đo động xử lý sau (PPK) có nhiều ưu điểm trong công tác đo vẽ thành lập bản đồ số. Công tác xây dựng lưới khống chế được giảm đáng kể. Việc đo chi tiết không đòi hỏi sự thông hướng giữa điểm khống chế với điểm chi tiết như phương pháp đo vẽ truyền thống bằng máy toàn đạc điện tử. Thời gian đo chi tiết và số nhân công ít hơn. Theo một số nghiên cứu cho thấy khi sử dụng phương pháp RTK trong đo vẽ bản đồ tỷ lệ lớn có thể giảm $50 \%-60 \%$ chi phí nhân lực, giảm tới 50\% giá thành công trình. Tuy vậy RTK vẫn có những hạn chế nhất định như hạn chế về khoảng cách chuyền tín hiệu liên tục từ trạm tham chiếu (trạm Base) đến trạm đo đạc (Rover). Trong khi đó, phương pháp đo động xử lý sau (PPK) có thể khắc phục vấn đề trên của phương pháp RTK. Tuy nhiên phương pháp PPK cũng có hạn chế là không kiểm soát được độ chính xác xác định vị trí tại thời điểm đo đạc và độ chính xác suy giảm khi tăng dần khoảng cách tư Base đến Rover. Trong nội dung nghiên cứu này, chúng tôi đi sâu khảo sát độ chính xác đo xác định tọa độ các điểm chi tiết khi thành lập bản đồ số tỷ lệ lớn bằng công nghệ RTK từ trạm Base và tham chiếu hoạt động liên tục Cors. Vấn đề kết hợp công nghệ đo RTK với đo tọa độ một số điểm GPS thông qua bài toán biến đổi tọa độ nhằm nâng cao độ chính xác trong thành lập bản đồ số tỷ lệ lớn.

\section{Khảo sát độ chính xác đo chi tiết bằng công nghệ RTK.}

\section{a. Phưong án đo tù̀ trạm Base:}

Để xem xét độ chính xác đo chi tiết bằng công nghệ RTK, chúng tôi thành lập lưới khống chế mặt bằng 4 điểm gốc và 9 điểm xác định bằng máy toàn đạc điện tử Trimble $\mathrm{S} 6$ và máy Trimble R7 GNSS. Độ chính xác lưới mặt bằng đo được sau bình sai đạt đường chuyền cấp 1 (Xem Hinh 1)

Bằng phương pháp đo RTK, tiến hành đo xác định lại tọa độ các điểm khống chế, tại mỗi điểm được đo 10 lần bằng máy Trimble R7 GNSS. Như vậy tọa độ các điểm khống chế được xem là trị thực so với điểm đo RTK. Áp dụng phương pháp đánh giá độ chính xác kết quả đo theo sai số thực theo công thức Bessen khi đo nhiều lần một đại lượng tại từng điểm khống chế và tổng hợp toàn lưới [1]. Các kết quả nêu ở bảng 1 . (Xem Bảng 1) 


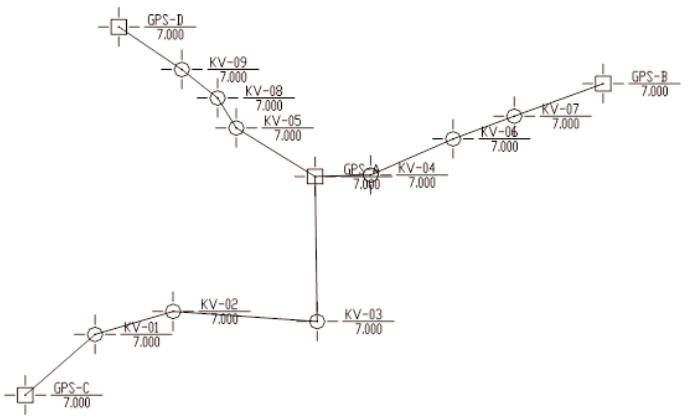

Hình 1: So đồ lưới khống chế đường chuyền cấp 1

Để kiểm chứng độ chính xác kết quả đo RTK, chúng tôi bố trí đo chi tiết thành lập bản đồ số địa hình tại khu vực công viên Hòa Bình. Các điểm chi tiết được đo hai lần bằng máy toàn đạc điện tử và công nghệ RTK. Bằng công thức đánh giá độ chính xác dãy kết quả trị đo kép [1], chúng tôi có được kết quả nêu ở bảng 2 . Độ chính xác vị trí điểm chi tiết về mặt bằng và độ cao đạt $4 \mathrm{~cm}$. (Xem Bảng 2)

b. Phương án đo tù̀ trạm tham chiếu hoạt động liên tục CORS:
Hiện nay công nghệ RTK ứng dụng trạm tham chiếu hoạt động liên tục CORS đang được ứng dụng nhiều trong thực tế sản xuất. Để phân tích độ chính xác của phương pháp này chúng tôi tiến hành khảo sát đo lại các điểm khống chế đã có tọa độ làm trị thực tại 3 vị trí đặt trạm CORS gồm: Trường Đại học Mỏ-Địa chất, Hà Đông Hà Nội và TP.Hải Dương. Độ chính xác nhận được như sau: (Xem Bảng 3, 4, 5)

Từ kết quả trên chúng ta thấy rằng độ chính xác xác định điểm chi tiết từ các trạm Cors phụ thuộc vào khoảng cách từ điểm đo đến vị trí trạm Cors. Khoảng cách đo chi tiết tốt nhất giữa trạm Cors và Rove nhỏ hơn $10 \mathrm{~km}$.

\section{Kết hợp công nghệ RTK với đo GPS}

Do đặc điểm của công nghệ RTK, độ chính xác các điểm đo phụ thuộc vào độ chính xác điểm trạm Base và khoảng cách giữ tram Base và Rove. Công nghệ RTK khi đo vẽ bản đồ khu vực có địa vật che khuất sẽ không thực hiện được. Đối với khu vực có cây tán lớn, các công trình xây dựng... che khuất, số lượng điểm chi

Bảng 1: Đánh giá độ chính xác đo RTK

\begin{tabular}{|c|c|c|c|c|c|c|}
\hline \multirow{2}{*}{ Tên điểm kiểm tra } & \multicolumn{2}{|c|}{ Đánh giá theo công thức sai số thực } & \multicolumn{3}{c|}{ Đánh giá theo công thức Bessen } \\
\cline { 2 - 7 } & $\mathrm{m}_{\mathrm{x}(\mathrm{m})}$ & $\mathrm{m}_{\mathrm{y}(\mathrm{m})}$ & $\mathrm{m}_{\mathrm{d}(\mathrm{m})}$ & $\mathrm{m}_{\mathrm{x}(\mathrm{m})}$ & $\mathrm{m}_{\mathrm{y}(\mathrm{m})}$ & $\mathrm{m}_{\mathrm{d}(\mathrm{m})}$ \\
\hline GPS-A & 0.01282 & 0.01947 & 0.02331 & 0.00084 & 0.00182 & 0.00200 \\
\hline GPS-B & 0.03561 & 0.03435 & 0.04948 & 0.00089 & 0.00356 & 0.00367 \\
\hline$\ldots \ldots$. & & & & & & \\
\hline KV-2 & 0.03883 & 0.02465 & 0.04599 & 0.00164 & 0.00167 & 0.00235 \\
\hline KV-8 & 0.02232 & 0.02046 & 0.03028 & 0.00259 & 0.00485 & 0.00550 \\
\hline KV-9 & 0.00261 & 0.02656 & 0.02669 & 0.00292 & 0.00329 & 0.00439 \\
\hline Tổng hợp Toàn lưới & 0.02467 & 0.02064 & 0.03217 & 0.00203 & 0.00354 & 0.00420 \\
\hline
\end{tabular}

Bảng 2: Độ chính xác đo RTK theo công thức trị đo kép

\begin{tabular}{|c|c|c|c|c|c|c|c|}
\hline \multirow{2}{*}{ TT } & \multicolumn{3}{|c|}{ Tọa độ điểm chi tiết (RTK) } & \multicolumn{4}{|c|}{ Hiệu tọa độ giữa hai phương pháp } \\
\hline & $\mathrm{X}$ & $\mathrm{Y}$ & $\mathrm{H}$ & $\mathrm{dx}$ & dy & $\overline{\mathrm{dd}}$ & $\mathrm{dh}$ \\
\hline 1 & 2330107.532 & 581618.369 & 6.210 & -0.135 & 0.077 & 0.155 & -0.140 \\
\hline 2 & 2330107.532 & 581618.369 & 6.210 & -0.133 & 0.077 & 0.154 & -0.150 \\
\hline 3 & 2330107.532 & 581618.372 & 6.210 & -0.135 & 0.080 & 0.157 & -0.140 \\
\hline \multicolumn{8}{|l|}{....... } \\
\hline 169 & 2330107.287 & 581637.208 & 6.210 & -0.130 & 0.046 & 0.138 & -0.140 \\
\hline 170 & 2330107.642 & 581657.425 & 6.230 & -0.142 & 0.070 & 0.158 & -0.160 \\
\hline 171 & 2330107.642 & 581657.425 & 6.230 & -0.116 & 0.060 & 0.131 & -0.110 \\
\hline \multicolumn{4}{|c|}{$m=\operatorname{spr}([d d] / 2 n)$} & 0.032 & 0.022 & 0.039 & 0.045 \\
\hline
\end{tabular}


Bảng 3: Đánh giá độ chính xác đo RTK-trạm Cors Truờng Mỏ-Địa chất (3 km)

\begin{tabular}{|c|c|c|c|c|c|c|c|c|}
\hline \multirow{2}{*}{$\begin{array}{c}\text { Điểm kiểm } \\
\text { tra }\end{array}$} & \multicolumn{3}{|c|}{ Đánh giá theo công thức sai số thực } & \multicolumn{3}{c|}{ Đánh giá theo công thức Bessen } \\
\cline { 2 - 9 } & $\mathrm{m}_{\mathrm{x}(\mathrm{m})}$ & $\mathrm{m}_{\mathrm{y}(\mathrm{m})}$ & $\mathrm{m}_{\mathrm{d}(\mathrm{m})}$ & $\mathrm{m}_{\mathrm{H}(\mathrm{m})}$ & $\mathrm{m}_{\mathrm{x}(\mathrm{m})}$ & $\mathrm{m}_{\mathrm{y}(\mathrm{m})}$ & $\mathrm{m}_{\mathrm{d}(\mathrm{m})}$ & $\mathrm{m}_{\mathrm{H}(\mathrm{m})}$ \\
\hline GPS-A & 0.0119 & 0.0250 & 0.0281 & 0.0168 & 0.0016 & 0.0023 & 0.0028 & 0.0149 \\
\hline GPS-B & 0.0334 & 0.0404 & 0.0526 & 0.0117 & 0.0036 & 0.0041 & 0.0054 & 0.0080 \\
\hline GPS-C & 0.0284 & 0.0358 & 0.0465 & 0.0055 & 0.0034 & 0.0026 & 0.0043 & 0.0091 \\
\hline GPS-D & 0.0301 & 0.0360 & 0.0470 & 0.0102 & 0.0021 & 0.0031 & 0.0037 & 0.0055 \\
\hline T.Bình & $\mathbf{0 . 0 2 6 0}$ & $\mathbf{0 . 0 3 4 3}$ & $\mathbf{0 . 0 4 3 6}$ & $\mathbf{0 . 0 1 1 1}$ & $\mathbf{0 . 0 0 2 7}$ & $\mathbf{0 . 0 0 3 0}$ & $\mathbf{0 . 0 0 4 1}$ & $\mathbf{0 . 0 0 9 4}$ \\
\hline
\end{tabular}

Bảng 4: Đánh giá độ chính xác đo RTK-trạm Cors Hà Đông - TP Hà Nội $(15 \mathrm{~km})$

\begin{tabular}{|c|c|c|c|c|c|c|c|c|}
\hline \multirow{2}{*}{$\begin{array}{c}\text { Điểm kiểm } \\
\text { tra }\end{array}$} & \multicolumn{3}{|c|}{ Đánh giá theo công thức sai số thực } & \multicolumn{4}{c|}{ Đánh giá theo công thức Bessen } \\
\cline { 2 - 9 } & $\mathrm{m}_{\mathrm{x}(\mathrm{m})}$ & $\mathrm{m}_{\mathrm{y}(\mathrm{m})}$ & $\mathrm{m}_{\mathrm{d}(\mathrm{m})}$ & $\mathrm{m}_{\mathrm{H}(\mathrm{m})}$ & $\mathrm{m}_{\mathrm{x}(\mathrm{m})}$ & $\mathrm{m}_{\mathrm{y}(\mathrm{m})}$ & $\mathrm{m}_{\mathrm{d}(\mathrm{m})}$ & $\mathrm{m}_{\mathrm{H}(\mathrm{m})}$ \\
\hline GPS-A & 0.0484 & 0.0624 & 0.0790 & 0.0630 & 0.0205 & 0.0237 & 0.0314 & 0.0705 \\
\hline GPS-B & 0.0884 & 0.0701 & 0.1128 & 0.0400 & 0.0070 & 0.0205 & 0.0217 & 0.1106 \\
\hline GPS-C & 0.0764 & 0.0522 & 0.0926 & 0.0872 & 0.0509 & 0.0205 & 0.0549 & 0.1011 \\
\hline GPS-D & 0.1024 & 0.0968 & 0.1409 & 0.0881 & 0.0837 & 0.0712 & 0.1098 & 0.0853 \\
\hline T.Bình & $\mathbf{0 . 0 7 8 9}$ & $\mathbf{0 . 0 7 0 4}$ & $\mathbf{0 . 1 0 6 3}$ & $\mathbf{0 . 0 6 9 6}$ & $\mathbf{0 . 0 4 0 5}$ & $\mathbf{0 . 0 3 4 0}$ & $\mathbf{0 . 0 5 4 5}$ & $\mathbf{0 . 0 9 1 9}$ \\
\hline
\end{tabular}

Bảng 5: Đánh giá độ chính xác đo RTK- trạm Cors TP Hải Dưong (60 km)

\begin{tabular}{|c|c|c|c|c|c|c|c|c|}
\hline \multirow{2}{*}{$\begin{array}{c}\text { Điểm kiểm } \\
\text { tra }\end{array}$} & \multicolumn{3}{|c|}{ Đánh giá theo công thức sai số thực } & \multicolumn{4}{c|}{ Đánh giá theo công thức Bessen } \\
\cline { 2 - 9 } & $\mathrm{m}_{\mathrm{x}(\mathrm{m})}$ & $\mathrm{m}_{\mathrm{y}(\mathrm{m})}$ & $\mathrm{m}_{\mathrm{d}(\mathrm{m})}$ & $\mathrm{m}_{\mathrm{H}(\mathrm{m})}$ & $\mathrm{m}_{\mathrm{x}(\mathrm{m})}$ & $\mathrm{m}_{\mathrm{y}(\mathrm{m})}$ & $\mathrm{m}_{\mathrm{d}(\mathrm{m})}$ & $\mathrm{m}_{\mathrm{H}(\mathrm{m})}$ \\
\hline GPS-A & 0.2742 & 0.2188 & 0.3508 & 0.8948 & 0.0152 & 0.0082 & 0.0173 & 0.0102 \\
\hline GPS-B & 0.2336 & 0.1404 & 0.2772 & 0.5636 & 0.0044 & 0.0050 & 0.0067 & 0.5165 \\
\hline GPS-C & 0.3070 & 0.1980 & 0.3704 & 0.6419 & 0.0041 & 0.0113 & 0.0120 & 0.0571 \\
\hline GPS-D & 0.1174 & 0.0451 & 0.1258 & 0.1879 & 0.0205 & 0.0292 & 0.0357 & 0.0678 \\
\hline T.Bình & $\mathbf{0 . 2 3 3 1}$ & $\mathbf{0 . 1 5 0 6}$ & $\mathbf{0 . 2 8 1 1}$ & $\mathbf{0 . 5 7 2 1}$ & $\mathbf{0 . 0 1 1 1}$ & $\mathbf{0 . 0 1 3 4}$ & $\mathbf{0 . 0 1 7 9}$ & $\mathbf{0 . 1 6 2 9}$ \\
\hline
\end{tabular}

tiết đo được bằng RTK chỉ đạt 50-80\%. do vậy chúng ta phải dùng máy toàn đạc điện tử đo bổ xung các điểm còn lại. Thay cho việc xây dựng lưới khống chế đo vẽ để đo bổ xung các điểm chi tiết rải rác trên toàn bộ khu vực đo, chúng tôi đề xuất thực hiện nâng cao độ chính xác điểm đo RTK làm điểm trạm đo, đặt máy toàn đạc điện tử đo bổ xung các điểm chi tiết còn lại.

Để nâng cao độ chính xác điểm đo RTK, chúng ta bố trí đo một số điểm đã xác định bằng công nghệ RTK bằng phương pháp GPS tĩnh. Các điểm này có tọa độ từ công nghệ $\mathrm{RTK}$ và GPS được gọi là điểm song trùng. Thông qua bài toán biến đổi tọa độ trên mặt phẳng gồm: phép biến đổi Affine, Helmert, Biến đổi Đa thức [2], [3]. Các công thức biến đổi gồm:
a-Phép biến đổi Affine.

$$
\begin{aligned}
& \mathrm{x}_{2}=\mathrm{ax}_{1}+\mathrm{by}_{1}+\mathrm{c} \\
& \mathrm{y}_{2}=\mathrm{dx}_{1}+\mathrm{ey}_{1}+\mathrm{g}
\end{aligned}
$$

b- Phép biến đổi Helmert.

$$
\begin{aligned}
& \mathrm{x}_{2}=\mathrm{x}_{0}+\mathrm{ax}_{1}-\mathrm{by}_{1} \\
& \mathrm{y}_{2}=\mathrm{y}_{0}+\mathrm{bx}_{1}+\mathrm{ay}_{1}
\end{aligned}
$$

c-Phép biến đổi đa thức bậc 2 .

$$
\begin{aligned}
& \mathrm{x}_{2}=\mathrm{a}_{1}+\mathrm{b}_{1} \mathrm{x}_{1}+\mathrm{c}_{1} \mathrm{y}_{1}+\mathrm{d}_{1} \mathrm{x}_{1}{ }^{2}+\mathrm{e}_{1} \mathrm{y}_{1}{ }^{2}+\mathrm{g}_{1} \mathrm{x}_{1} \mathrm{y}_{1} \\
& \mathrm{y}_{2}=\mathrm{a}_{2}+\mathrm{b}_{2} \mathrm{x}_{1}+\mathrm{c}_{2} \mathrm{y}_{1}+\mathrm{d}_{2} \mathrm{x}_{1}{ }^{2}+\mathrm{e}_{2} \mathrm{y}_{1}{ }^{2}+\mathrm{g}_{2} \mathrm{x}_{1} \mathrm{y}_{1}
\end{aligned}
$$

Tùy theo số điểm có tọa độ song trùng, modul chương trình tự động xác định các hệ số biến đổi 
và tính lại tọa độ đo bằng RTK được hiệu chỉnh dựa vào tọa độ GPS. Sơ đồ khối và modul chương trình được nêu trong hình 2 và hình 3 .

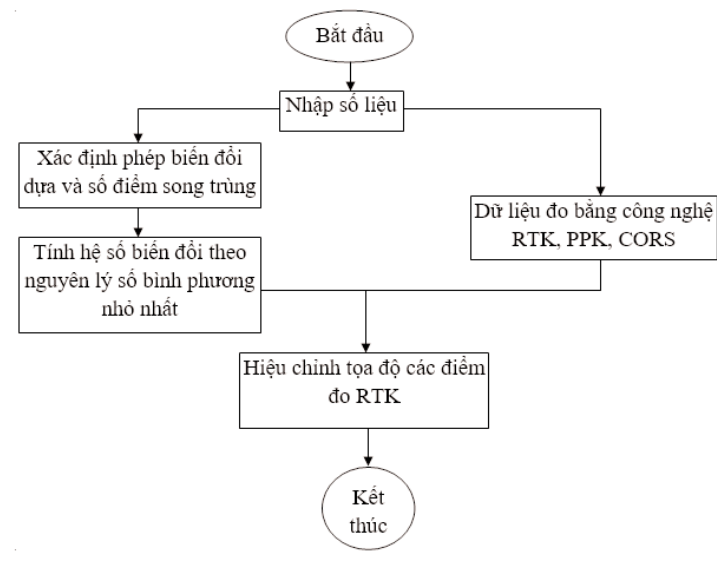

\section{Hình 2: So đồ khối chương trình}

Qua kết quả tính toán thực nghiệm cho thấy độ chính xác các điểm đo RTK được hiệu chỉnh dựa vào các điểm song trùng đã nâng cao độ chính xác. Với số điểm song trùng từ 3 đến 5 điểm, độ chính xác vị trí điểm $\left(\mathrm{m}_{\mathrm{d}}\right)$ đạt được từ $1 \mathrm{~cm}$ đến $4 \mathrm{~cm}$. Các kết quả tính toán được mô ta dưới đây:

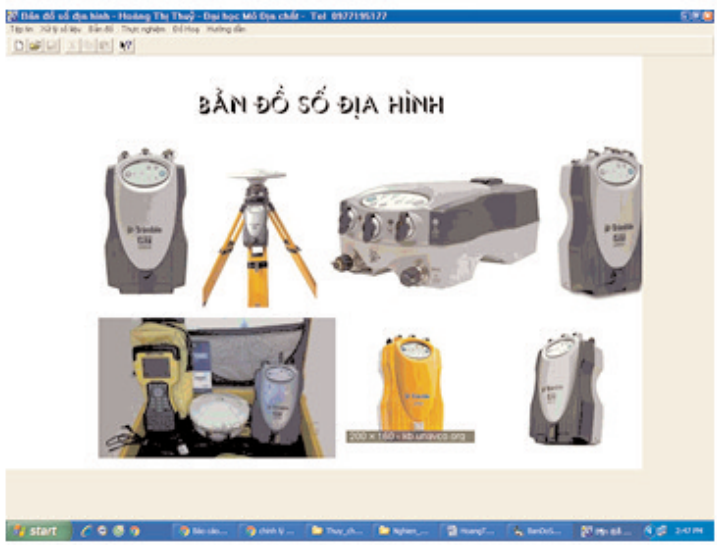

Hìn 3: Modul chwong trình

a- Tọa độ các điểm song trùng

1) GPS_D 2330690.979581501 .4138 .404 $2330690.911581501 .458 \quad 8.404$

2) GPS A 2330384.912581883 .3856 .735 $2330384.804 \quad 581883.443 \quad 6.735$

3) GPS_B 2330461.978 582152.043 7.163 2330461.889582152 .0947 .163

b- Hệ số tính chuyển

Bảng 6

\begin{tabular}{|c|c|c|c|c|c|c|c|}
\hline \multicolumn{2}{|c|}{ Tọa độ RTK } & \multicolumn{2}{c|}{ Tọa độ sau hiệu chỉnh } & \multicolumn{2}{c|}{ Chênh lệch tọa độ RTK } & \multicolumn{2}{c|}{ Chênh lệch tọa độ sau hiệu chỉnh } \\
\hline $\mathrm{x}$ & $\mathrm{y}$ & $\mathrm{x}$ & $\mathrm{y}$ & $\mathrm{dx}$ & $\mathrm{dy}$ & $\mathrm{dx}$ & $\mathrm{dy}$ \\
\hline 2330690.979 & 581501.417 & 2330690.909 & 581501.463 & 0.068 & -0.041 & -0.002 & 0.005 \\
\hline 2330690.987 & 581501.417 & 2330690.917 & 581501.463 & 0.076 & -0.041 & 0.006 & 0.005 \\
\hline 2330690.985 & 581501.415 & 2330690.915 & 581501.461 & 0.074 & -0.043 & 0.004 & 0.003 \\
\hline 2330545.543 & 581693.705 & 2330545.462 & 581693.753 & 0.096 & -0.033 & 0.015 & 0.015 \\
\hline 2330545.536 & 581693.704 & 2330545.455 & 581693.752 & 0.089 & -0.034 & 0.008 & 0.014 \\
\hline$\ldots$. & $\ldots .$. & $\ldots .$. & $\ldots \ldots$ & $\ldots \ldots$ & $\ldots .$. & $\ldots .$. & $\ldots .$. \\
\hline 2329937.896 & 581319.35 & 2329937.811 & 581319.373 & 0.08 & -0.019 & -0.005 & 0.004 \\
\hline 23329937.896 & 581319.349 & 2329937.811 & 581319.372 & 0.08 & -0.02 & -0.005 & 0.003 \\
\hline 2329937.897 & 581319.347 & 2329937.812 & 581319.37 & 0.081 & -0.022 & -0.004 & 0.001 \\
\hline 2329937.898 & 581319.352 & 2329937.813 & 581319.375 & 0.082 & -0.017 & -0.003 & 0.006 \\
\hline
\end{tabular}


Bảng 7

\begin{tabular}{|c|c|c|c|c|c|}
\hline \multicolumn{3}{|c|}{ Đánh giá theo công thức sai số thực } & \multicolumn{3}{c|}{$\begin{array}{c}\text { Đánh giá theo công thức sai số thực } \\
\text { Tọa độ đo RTK sau hiệu chỉnh } \\
\text { Tọa độ đo RTK }\end{array}$} \\
\hline $\mathrm{m}_{\mathrm{x}(\mathrm{m})}=0.085$ & $\mathrm{~m}_{\mathrm{y}(\mathrm{m})}=0.045$ & $\mathrm{~m}_{\mathrm{d}(\mathrm{m})}=0.076$ & $\mathrm{~m}_{\mathrm{x}(\mathrm{m})}=0.051$ & $\mathrm{~m}_{\mathrm{y}(\mathrm{m})}=0.028$ & $\mathrm{~m}_{\mathrm{d}(\mathrm{m})}=0.047$ \\
\hline
\end{tabular}

$\mathrm{A}=1.000161231223$

$\mathrm{B}=0.000024471837$

$\mathrm{C}=2330512.534666670000$

$\mathrm{D}=-0.000055222235$

$\mathrm{E}=0.999989785366$

$\mathrm{G}=581845.665000000000$

c- Sử dụng phép biến đổi Affine

$$
\begin{aligned}
& \mathrm{X} 2=\mathrm{A} * \mathrm{X} 1+\mathrm{B} * \mathrm{Y} 1+\mathrm{C} \\
& \mathrm{Y} 2=\mathrm{D} * \mathrm{X} 1+\mathrm{E} * \mathrm{Y} 1+\mathrm{G}
\end{aligned}
$$

d- Tọa độ các điểm đo RTK và tọa độ sau hiệu chỉnh. (Xem bảng 6) 7)

e- Kết quả đánh giá độ chính xác. (Xem bảng

\section{Kết luận:}

Trên cơ sở các kết quả nghiên cứu và thực nghiệm, chúng tôi thấy rằng việc ứng dụng công nghệ RTK kết hợp bài toán biến đổi tọa độ dựa vào điểm đo GPS trong công tác đo đạc thành lập bản đồ số địa hình, địa chính tỷ lệ lớn hoàn toàn đảm bảo độ chính xác yêu cầu theo quy định, đạt hiệu quả kinh tế cao. Modul chương trình tự động xác định các hệ số biến đổi và tính chuyển tọa độ đo RTK có ý nghĩa khoa học và thực tiễn. $\bigcirc$

\section{TÀI LIỆ THAM KHẢO}

[1]. Hoàng Ngọc Hà, Trương Quang Hiếu. 1999. Cơ sở toán học xử lý số liệu trắc địa. Nhà xuất bản Giao thông vận tải. Hà Nội

[2]. Hoàng Ngọc Hà. 2001. Tính toán trắc địa và cơ sở dữ liệu. Nhà xuất bản Giao thông vận tải. Hà Nội

[3]. Đinh Công Hòa. 2011. Lập trình bài toán trắc địa cơ sở. Nhà xuất bản Giao thông vận tải. Hà Nội. $\bigcirc$

\section{Summary}

\section{Improvement of accuracy of RTK-measured coordinates for large scale mapping}

Hoang Thi Thuy

\section{Hanoi University of Mining and Geology}

This study assessed accuracy of RTK for large scale mapping. In order to improve the accuracy of the map for mining tasks, RTK was integrated with statistic GPS. A programmed module was created to adjust RTK-measured coordinates based on statistic-GPS coordinates and by transforming the coordinates. The results showed that the integration of RTK and statistic GPS meets the accuracy and efficiency requirements the large scale mapping. $\mathrm{O}$ 